\title{
CANNON POSITION CONTROL THROUGH ELECTROMAGNETIC FIELDS, WITH TWO DEGREES OF FREEDOM
}

\author{
${ }^{1}$ John Castillo, ${ }^{2}$ Nathalia Gama, ${ }^{3}$ Dario Amaya \\ Virtual Applications Group-GAV, "Universidad Militar Nueva Granada-UMNG” \\ ${ }^{1}$ u1801647, ${ }^{2}$ gav, ${ }^{3}$ dario.amaya@unimilitar.edu.co
}

Summary: This paper presents the control of shot position of cannon driven by electromagnetic fields. First, the design and construction of a mechanical structure for the shot platform with two degrees of freedom is presented, then the designed and implementation of position control for shot of the cannon is presented. Finally, its present's adaptation of an inertial navigation system, which allow find the fall position of the shot projectile. For the designed of controller was used the root locus method, to obtain the desired response of the closed loop system from its open loop behavior. As a result, a response controlled was obtained; within the design, parameters predefined which allow control the position and orientation of the electromagnetic cannon shot.

Keywords: Electromagnetic cannon, closed loop control, inertial navigation system, armament.

\section{INTRODUCTION}

The cannon driven by electromagnetic fields allows the conversion of electromagnetic energy into kinetic energy to achieve the accelerated movement of a projectile along a defined path. Some of the advantages presented by electromagnetic field cannons are that they can accelerate projectiles at very high speeds, as the shot process is practically free of friction between the projectile and the armature, the load can be easily installed and the system in general, has a high-energy conversion efficiency [1]. The principle of operation of the coil cannon is based on the law of electromagnetic effect formulated by Carl Friedrich, which can be studied in [2], [3].

This article shows the methodology and the results obtained with the implementation of a system of control of orientation and elevation of cannon driven by electromagnetic fields. The implementation of this system will allow the automation of the positioning of the cannon using not only the control system in closed loop, but also using a GPS and a compass and IMU of 6 axes, obtaining in this way an entire inertial navigation system. As explained in [4], inertial navigation systems (INS) combine IMU inertial sensors widely used in navigation and guidance systems, such as accelerometers and gyroscopes, to a platform with a common reference system, for the generation of data required for the inertial navigation.

As evidenced in [5], the use of inertial navigation systems in the military industry has presented several applications in the last years, from the acquisition of launching coordinates of cannon type Obus M-101, with transmission [6], to their applications in aeronautics [7].

\section{METHODOLOGY}

The methodology consists of two main parts shown below.

The first part corresponds to the design and construction of the shot platform. The second part corresponds to the implementation of the closed-loop control system and the implementation of the inertial navigation system. Of previous works, in which a prototype of cannon-coil of a stage was implemented, there are two coils of copper with a plastic tube as armor, with a mass $=265.19$ grams and with the center of mass located in the geometric center of tube. The CAD model of the coils and the armor are shown in Figure 1.

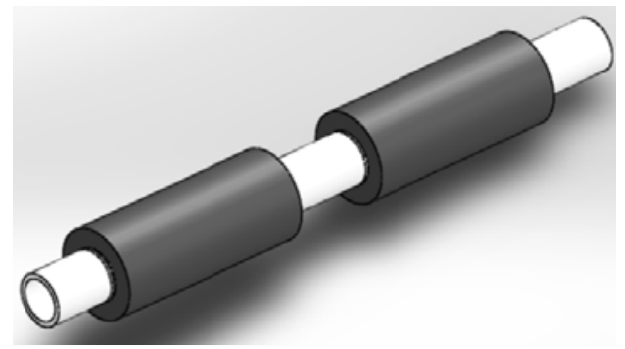

Figure 1. Coils and armature of the electromagnetic cannon. Source: Authors. 
Starting from the existing coils, a mechanical platform with two degrees of freedom is designed and constructed, allowing the control of its horizontal and vertical angle. The platform positioning automation is done through of a computer interface using of Arduino development cards. The shot platform is built in aluminum and it uses servomotors with Torque $13.2 \mathrm{~kg}-\mathrm{cm}$ at $6 \mathrm{~V}$. The design also uses a transmission chain in two axes; this reduces the complexity of the design and gives the required robustness. The design of the shot platform and the platform once implemented, are observed in

Figure 2.

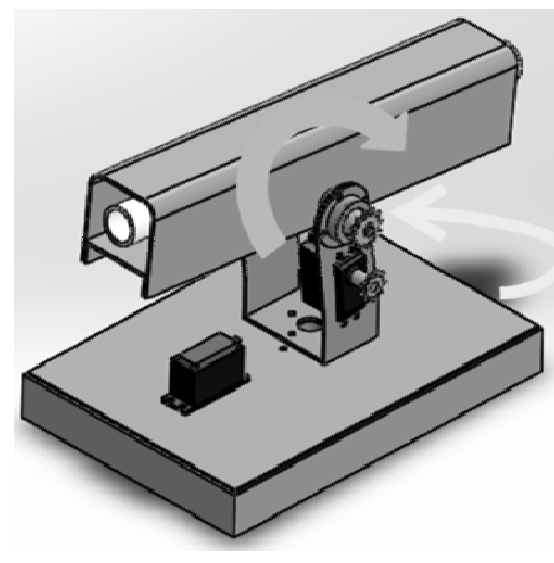

(a)

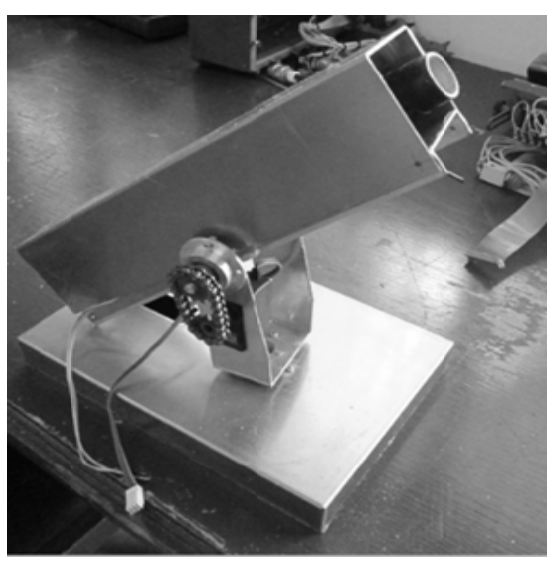

(b)

Figure 2. Design for the automation of pitch and yaw position control. (a) Platform in CAD. (b) Real platform implemented. Source: Authors.

\section{Electronic schematic}

The design considers the connections between the computer and the Arduino board, which is fed by the sensors information and allows the output of the control signal to the servomotors, as shown in Fig. 3. A brief explanation of the hardware used for the implementation of the controller and the communication system is provided below.

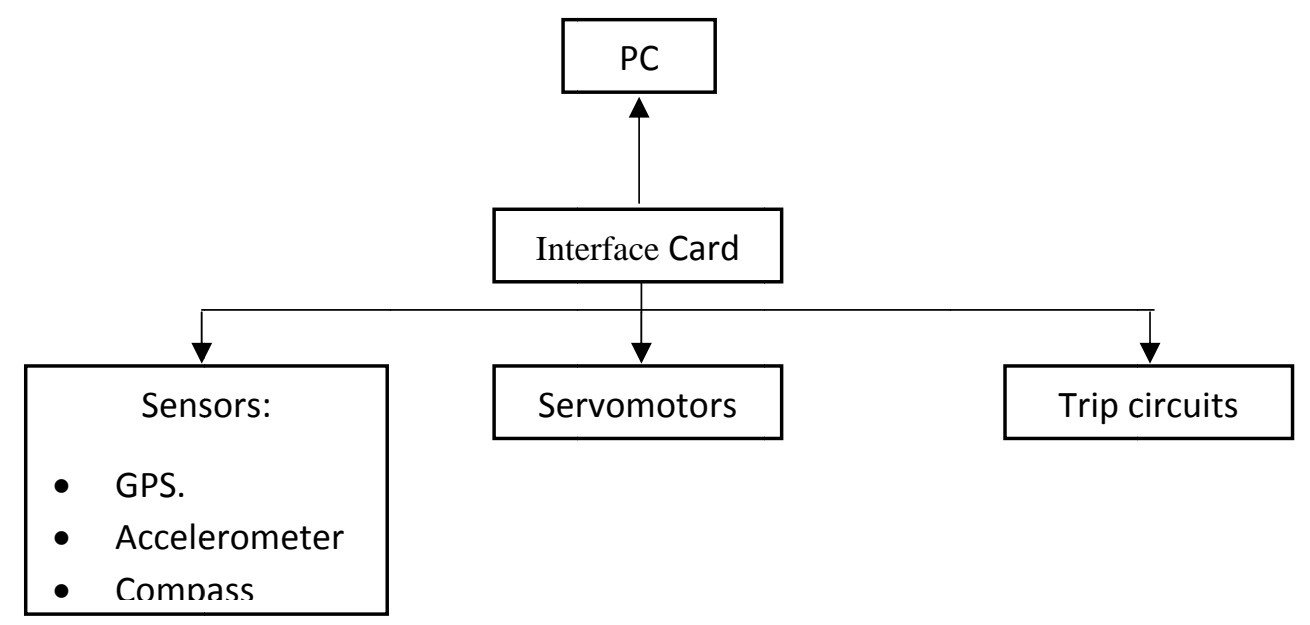

Figure 3. Connection blocks diagram.Source:Authors.

The acquisition of the information provided by the sensors is done through an Arduino Leonardo card, which consists of an ATmega32U4 micro-controller and handles communication protocols such as SPI, I2C and serial. Its connectivity to the computer is made via the USB port converted to serial. One of the sensors used is an electronic compass with tilt compensation, which uses a 3-axis magnetometer and a 3-axis accelerometer; these signals are processed to compensate errors generated by tilts.

The geographic location is made through a Venus GPS receiver, can be configured with a refresh rate of up to $10 \mathrm{~Hz}$ to recover the NEMA chain with 14 satellite tracking channels, this port has a UART and SPI port. IMUS are systems that integrate accelerometers and gyroscopes on a single board, in this case the gyroscope, IMU3000 two-axis, LY530ALH of a shaft and the ADXL345 accelerometer of three axes, resulting in an IMU of 6 degrees. All the instrumentation allows to read the data acquired through the communication ports and have 
the advantage of already having linear data, facilitating the implementation and simplicity in the control algorithm.

\section{Communication system}

The communication with the completely sensory was mounted through the available Arduino card ports, implemented in the communication scheme shown in Figure 4. This communication strategy allows obtaining all the sensory data using the SPI communication protocol and Alternating their reading between the GPS and the IMU, which are the ones that have such hardware. Furthermore, the electronic compass is communicated through the I2C protocol and finally the control output would be implemented by regulating the useful cycle in a PWM signal.

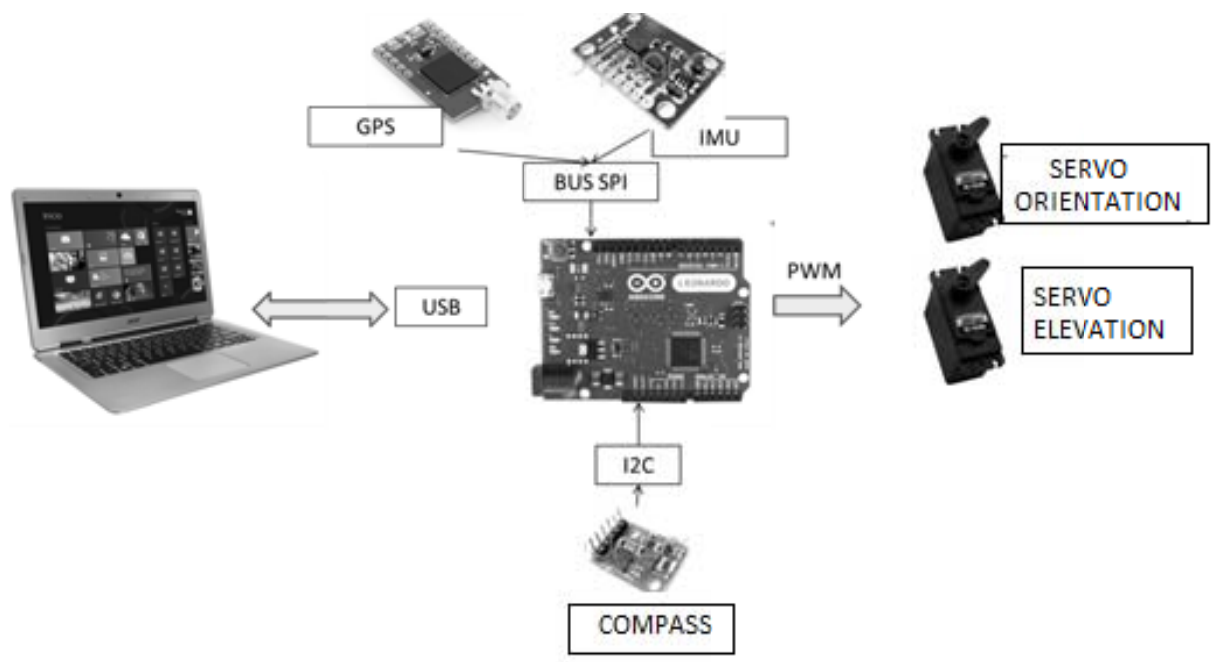

Figure 4. Communications Scheme. Source: Authors.

Graphic interface

A simple interface is designed with the Microsoft Visual C \# 2010 compiler, in which you can visualize the coordinates of the device as well as the current orientation and elevation, as well as the desired orientation and elevation. The user interface is shown in Figure 5.

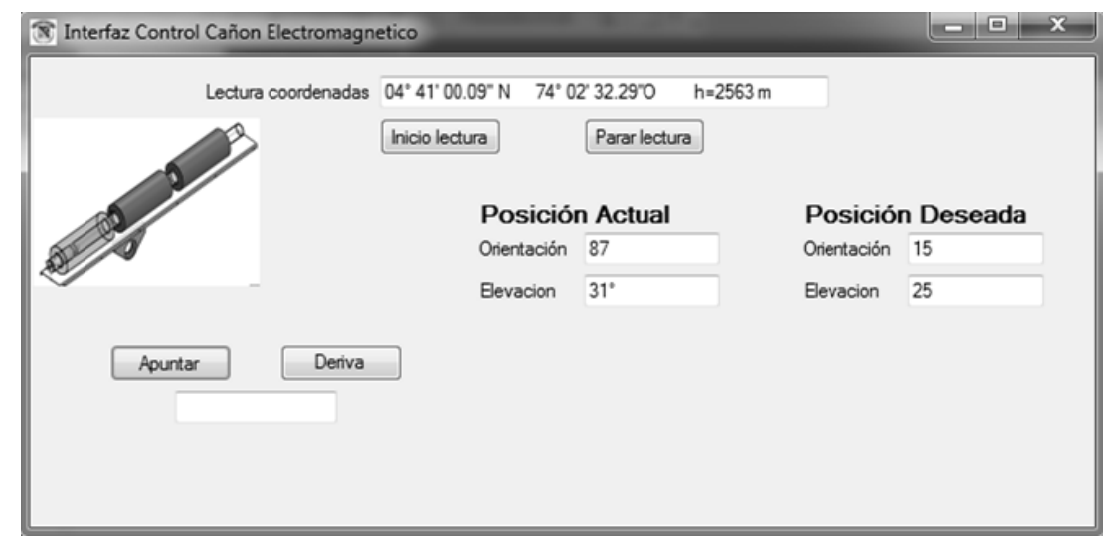

Figure 5. User Interface. Source:Authors.

Control in position by root location

The main idea of control design by the root locus method is to find the system response to closed loop from the diagram of the place of roots to open loop. This will modify the closed loop response by adding zeros and / or poles to the original plant. The intention is to implement a strategy of digital control by place of the roots. The objective is that a reference value be established for each axis and that the servomotor reaches that value, regardless of whether there may be position disturbances. The strategy for the control design proposed shown below:

1. Determine the mathematical model of the servomotor.

2. It will be modelled continuously, and later converted into discrete time.

3. The open loop response will be simulated 
4. The design will be simulated by place of the roots

5. After checking in simulations, the control is implemented in the cannon.

\section{RESULTS}

Below the design of the controller and its implementation in the plant is shown. The root locus method requires in first place the identification of the plant to find the open loop response. The control scheme for the respective plant is shown in Figure 6. (Hernández 2007).

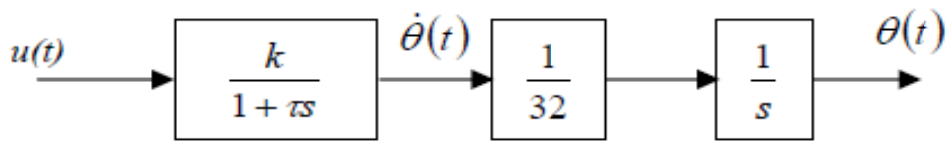

Figure 6. Control scheme in open loop. Source: Authors.

The transfer function of the plant was mathematically characterized by the response obtained from the sensor information, after the application of the PWM pulse generated by the computer through the Arduino Leonardo card. In Figure $6 u(t)$ is identified as the reference voltage of the pulse PWM $\dot{\theta}(t)$ is the voltage proportional to the angular velocity and finally $\theta(t)$, is the voltage proportional to the position. In the scheme a gain of $1 / 32$ is introduced which is intended to represent the reduction of the servomotor [8], [9].

In the plant block, the transfer function $G(s)=\frac{k}{1+T s}$ is identified where $\mathrm{k}$ is the system gain and is obtained by multiplying the time constant $\mathrm{T}$, which is obtained from the elapsed time since it starts the step up to $62 \%$ by the final value reached by the output signal. As shown in Fig. 7, the field-controlled DC motor modeling scheme was performed, wherein the armature current is constant. The parameters obtained from the motor were as shown in Figure 7.

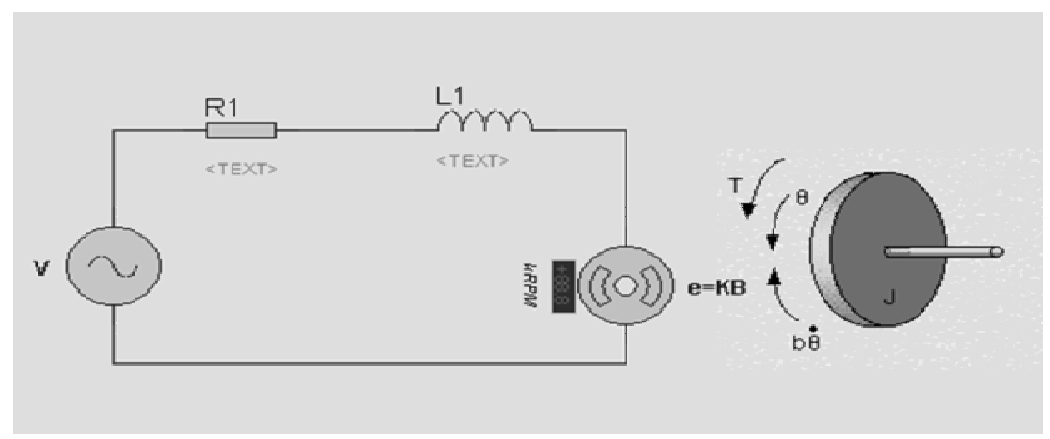

Figure 7. DC motor modeling scheme. Source: Authors.

Table 1. Parameters of DC motor.

\begin{tabular}{|l|l|}
\hline \multicolumn{1}{|c|}{ Parameter } & \multicolumn{1}{c|}{ Value } \\
\hline Moment of inertia of the rotor (J) & $4.22 \mathrm{e}-6 \mathrm{~kg} \cdot \mathrm{m}^{\wedge} 2 / \mathrm{s}^{\wedge} 2$ \\
\hline Mechanical system damping coefficient (b) & $4.5077 \mathrm{E}-6 \mathrm{Nms}$ \\
\hline Electromotive force constant (K=Ke=Kt) & $1.0274 \mathrm{Nm} / \mathrm{Amp}$ \\
\hline Electrical resistance (R) & 5 ohm \\
\hline Electrical Inductance (L) & $3.75 \mathrm{E}-6 \mathrm{H}$ \\
\hline Input (V) & Source Tension \\
\hline Output (theta): & $\begin{array}{l}\text { Position of the shaft (the rotor and shaft are } \\
\text { considered rigid) }\end{array}$ \\
\hline
\end{tabular}

According to the parameters shown in Table 1, and the combination of Newton's laws next to Kirchhoff's law, we get Eq. (1):

$$
L \frac{d i}{d t}+R i=V-K \dot{\theta}
$$

From where the transfer function shown in equation (2) must be obtained.

$$
\frac{\dot{\theta}}{\boldsymbol{V}}=\frac{\boldsymbol{K}}{\left.\boldsymbol{s}(\boldsymbol{J} s+\boldsymbol{b})(\boldsymbol{L} s+R)+\boldsymbol{K}^{2}\right)}
$$

And by choosing as state variables the armature current, motor speed and motor position you can write the state equations in matrix form, as shown in equation (3), and the output equation, shown in (4): 


$$
\begin{gathered}
\frac{d}{d t}\left[\begin{array}{c}
\theta \\
\dot{\theta} \\
i
\end{array}\right]=\left[\begin{array}{ccc}
0 & 1 & 0 \\
0 & -\frac{b}{J} & \frac{K}{J} \\
0 & -\frac{K}{L} & -\frac{R}{L}
\end{array}\right]\left[\begin{array}{c}
\theta \\
\dot{\theta} \\
i
\end{array}\right]+\left[\begin{array}{c}
0 \\
0 \\
1 \\
\frac{1}{L}
\end{array}\right] V \\
y=\left[\begin{array}{lll}
1 & 0 & 0
\end{array}\right]\left[\begin{array}{c}
\theta \\
\dot{\theta} \\
i
\end{array}\right]
\end{gathered}
$$

The following are the variables that were established as design parameters for the controller: (a) Stationary state error equal to zero, (b) sampling time (Ts) equal to $40 \mathrm{~ms}$, and (c) ) the maximum peak is less than $16 \%$. Figure 8 shows the system response in open loop.

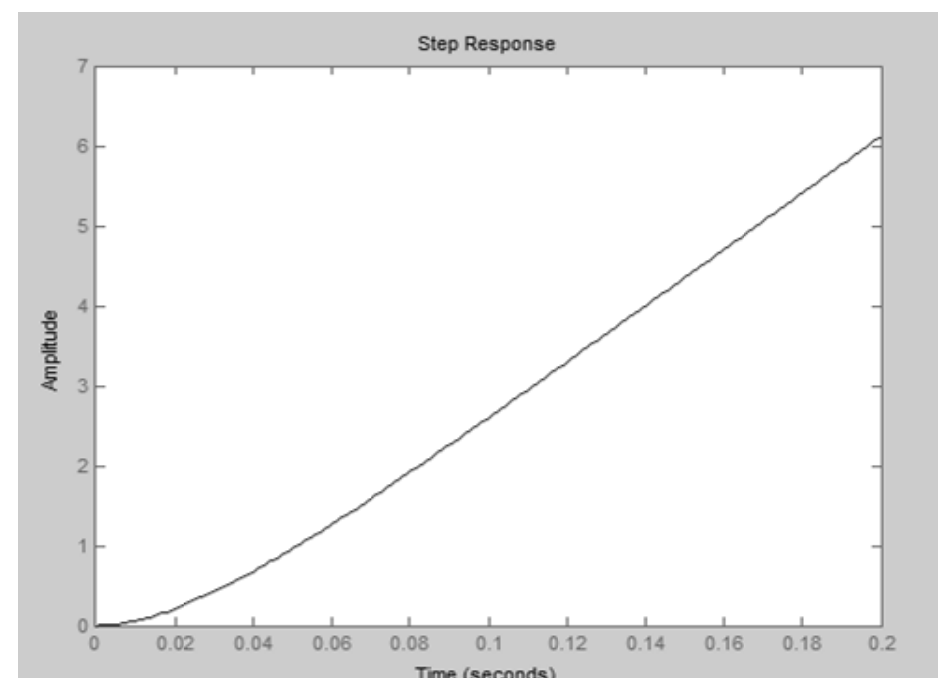

Figure 8. System response in open loop. Source: Authors.

For the root locus design, Matlab was used to convert the continuous transfer function to discrete with the command c2dm, obtaining the function of transfer of the output in discrete time to the input voltage, as shown in equation (5).

$$
\frac{\theta(z)}{V(z)}=\frac{0.9538 Z+0.0195}{Z^{2}-z}
$$

Figure 9 shows a simulation of the closed-loop system response before applying the controller.

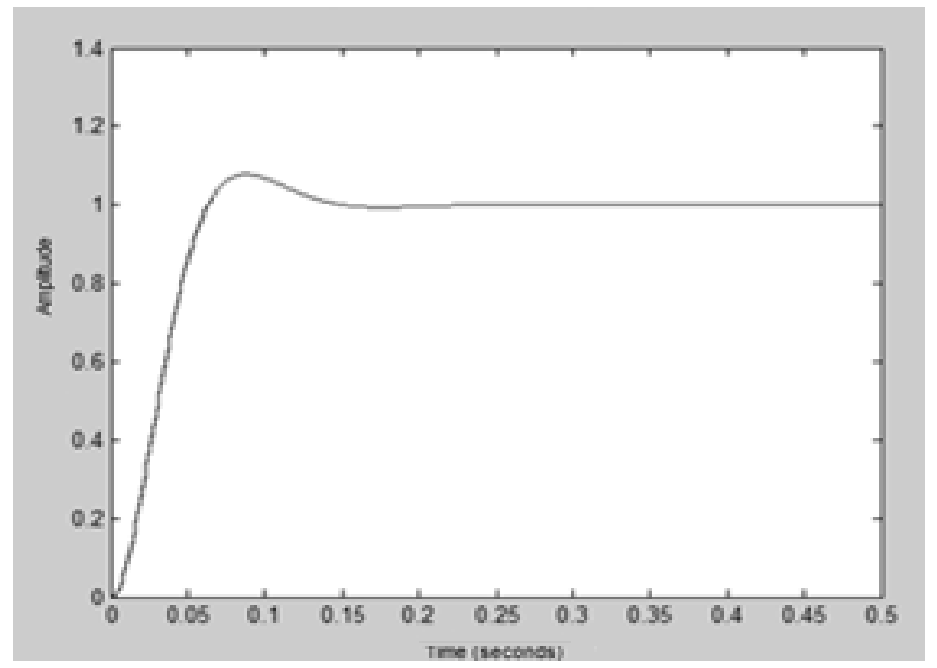

Figure 9. Closed loop response without controller. Source: Authors.

The intention of the root locus design is to obtain the closed-loop response from the open-loop root location diagram, which is achieved by adding poles to the first system and, in turn, by modifying the location of the roots and obtaining a new response in closed loop. 
To achieve zero steady state from the closed loop response, an integral control is implemented in continuous is 1 / $\mathrm{s}$, and an approximation is made by means of backward differences for the mapping of the $\mathrm{S}$ to $\mathrm{Z}$ plane.

This made it possible to determine that it was necessary to add a 1-pole at the root site. Using the Zgrid command of Matlab, the desired region was found, and a damping coefficient of 0.5 was set as the input parameter, obtaining the response shown in Figure 10 (b).
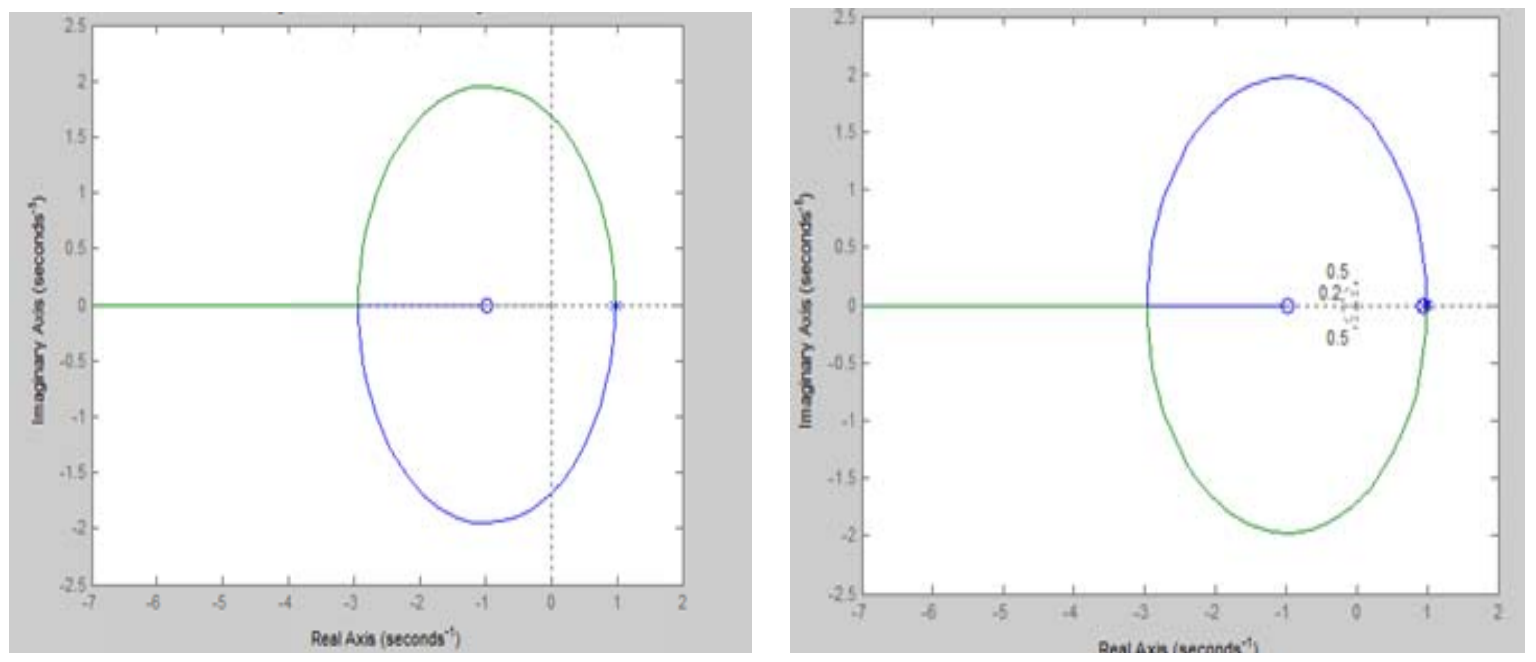

Figure 10. Place of roots. (A) In the original system. (B) On the system after the controller is implemented.

Since the system is unstable with any gain, to stabilize it takes the place of the roots to the unit circle, near the damping coefficient, and with movements in the poles and zeros of the system, based on trial and error stability is achieved Of the system and the desired response, shown in Figure 11.

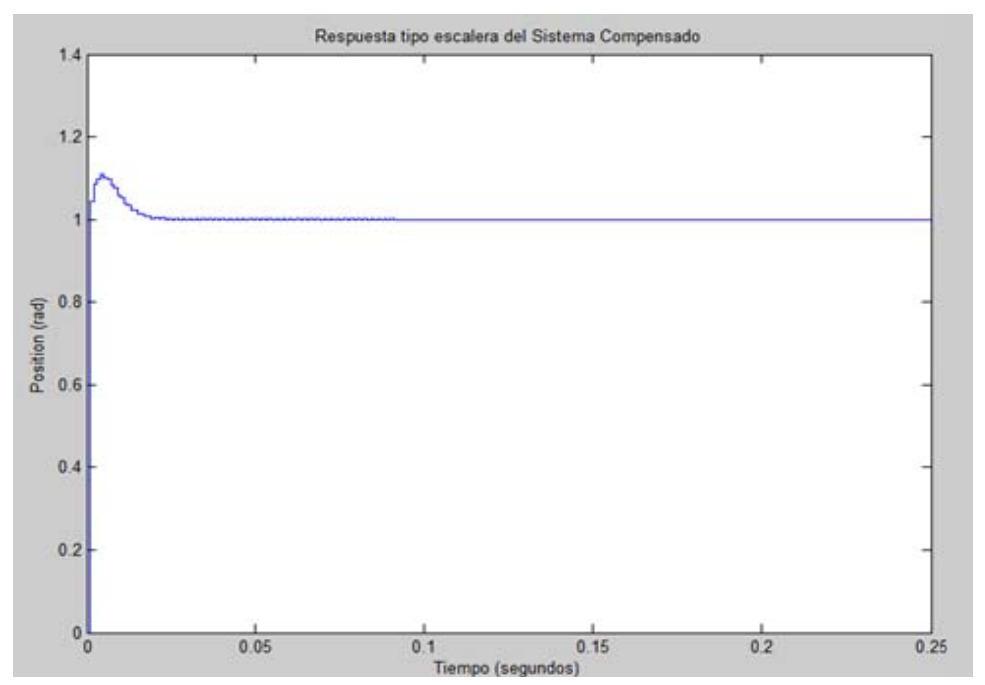

Figure 11. Response to step in closed loop.

Implementing the controller

The position control was performed correctly, finding inadequacies in the control in orientation, due to the strong magnetic fields generated at the moment of the shot. The position control was strongly affected by the sampling and processing times, thus affecting the response of the servo motors.

\section{CONCLUSIONS}

Was designed and implemented the mechanical structure for a shot platform based on load specifications and corroborating the designs with CAD simulations.

The mechanical structure of the system must be designed with light materials such as aluminum, as well as the simplicity of power transmission systems. The servo motors, although they already comprise an immersed control, must be modeled again taking into account the loads of the real plant in order to get closer to the desired result. 
The monitoring software should contemplate the current position and desired position giving the user a friendly and easy to use environment. The position control system for the cannon firing was designed and implemented in discrete time using the place method in the roots.

By means of a interface in C \#, the software of monitoring of firing for the cannon operated by means of electromagnetic fields was implemented.

\section{REFERENCES}

[1] Tao, Z., Wei, G., Zhiqiang, D., Yanhui, C., Mingtao, L. y Xiaochao, S. (2012). Experimental results from a 4-stage synchronous induction coilgun. 16th International Symposium on Electromagnetic Launch Technology (EML), 1-5.

[2] Sears, F.W., Zemansky, M. W., Young, H.D. y Freedman, R. (2009). Física Universitaria. México. Pearson.

[3] Serway, R.A. y Jewett J.H. (2009). Física para ciencias e ingeniería con Física Moderna. México. Cengage Learning.

[4] Hernández Miguel (2007). Control en Posición de un Servomotor de CC, Escuela Politécnica Superior de Elche, Facultad Ingeniería Industrial, España 12-29.

[5] Castillo, J., Amaya D., Avilés, S., Barrera, J., Moreno, D., Gutiérrez, J. (2011). Diseño e implementación de una plataforma de ubicación geográfica de los cañones tipo OBUS M-101, Ciencia y Tecnología del Ejército, 2 (2), 26-31.

[6] Sheard, K.; Scaysbrook, I.; Cox, D. (2008) MEMS sensor and integrated navigation technology for precision guidance, Position, IEEE Symposium on Location and Navigation, 1145,1151.

[7] Winkler, S., Buschmann, M., Kruger, L., Schulz, H.-W., Vorsmann, P. (2005). Multiple Sensor Fusion for Autonomous Mini and Micro Aerial Vehicle Navigation, IEEE TENCON 10, pp.1-6.

[8] Gómez Fernando, Modelado de Sistemas en Tiempo Continuo (2007), España Dinámica de Sistemas 2.1 Dpto. de Ingeniería Electrónica, de Sistemas Informáticos y Automática Universidad de Huelva, 10-22.

[9] Pozo, D., Diseño y construcción de una plataforma didáctica para medir ángulos de inclinación usando sensores inerciales como acelerómetro y giroscopio. Proyecto previo a la obtención del título de ingeniero en electrónica y control, Escuela Politécnica Nacional, Facultad de ingeniería eléctrica y electrónica, Quito, Ecuador. 